\title{
Intraoperative versus Early Postoperative Intraperitoneal Chemotherapy after Cytoreduction for Colorectal Peritoneal Carcinomatosis: an Experimental Study
}

\author{
Yvonne L. B. Klaver, MD ${ }^{1,2}$, Thijs Hendriks, PhD $^{2}$, Roger M. L. M. Lomme ${ }^{2}$, Harm J. T. Rutten, PhD ${ }^{1}$, \\ Robert P. Bleichrodt, $\mathrm{PhD}^{2}$, and Ignace H. J. T. de Hingh, $\mathrm{PhD}^{1}$ \\ ${ }^{1}$ Department of Surgery, Catharina Hospital, Eindhoven, The Netherlands; ${ }^{2}$ Department of Surgery, Radboud \\ University Nijmegen Medical Centre, Nijmegen, The Netherlands
}

\begin{abstract}
Background. Perioperative intraperitoneal chemotherapy is used as an adjunct to cytoreductive surgery (CS) for peritoneal carcinomatosis (PC) in order to prolong survival. Worldwide, hyperthermic intraperitoneal chemotherapy (HIPEC), early postoperative intraperitoneal chemotherapy (EPIC), and combinations of the two are used. It remains unclear which regimen is most beneficial.

Methods. The rat colon carcinoma cell line CC-531 was injected into the peritoneal cavity of $80 \mathrm{WAG} / \mathrm{Rij}$ rats to induce PC. Animals were randomized into four treatment groups $(n=20)$ : CS only, CS followed by HIPEC (mitomycin $35 \mathrm{mg} / \mathrm{m}^{2}$ at $41.5^{\circ} \mathrm{C}$ ), CS followed by EPIC during 5 days (i.p. injection of mitomycin on day 1 and 5-fluorouracil on days 2-5), and CS followed by HIPEC plus EPIC. Primary outcome was survival.

Results. In rats treated with CS only, median survival was 53 days (95\% confidence interval (CI) 49-57 days). In rats treated with CS followed by HIPEC, survival was significantly $(P=0.001)$ increased (median survival 94 days, 95\% CI 51-137 days). In the group treated with EPIC after CS, 12 out of 20 rats were still alive at the end of the experiment ( $P<0.001$ as compared with CS only). In the group receiving both treatments, 11 rats died of toxicity, and therefore this group was not included in the survival analysis. Conclusions. Both EPIC and HIPEC were effective in prolonging survival. The beneficial effect of EPIC on survival seemed to be more pronounced than that of HIPEC.
\end{abstract}

(C) The Author(s) 2011. This article is published with open access at Springerlink.com

First Received: 28 March 2011; Published Online: 12 August 2011

Y. L. B. Klaver, MD

e-mail: yvonne.klaver@catharina-ziekenhuis.nl
Further research is indicated to evaluate and compare the possible benefits and adverse effects associated with both treatments.

The combination of cytoreductive surgery (CS) and perioperative intraperitoneal chemotherapy is the only available curative option for patients with peritoneal carcinomatosis (PC) from colorectal cancer. By now, it has been widely accepted as the treatment of choice for patients with limited peritoneal carcinomatosis who are fit for major surgery. The aim of this treatment is to radically remove all visible tumor deposits from the peritoneal cavity, and to eradicate residual microscopic disease by adjuvant application of intraperitoneal chemotherapy. Promising results have been shown by several centers, achieving median survival which compares favorably with the outcomes of patients treated with palliative care, in whom life expectancy is usually limited to approximately 6 months. ${ }^{1-5}$

The adjuvant intraperitoneal chemotherapy can be applied directly after cytoreduction under hyperthermic conditions (hyperthermic intraperitoneal chemotherapy, HIPEC) as a part of the surgical procedure, or can be started on the first postoperative day and continued for several (usually five) days (early postoperative intraperitoneal chemotherapy, EPIC). Both techniques are currently offered to patients with colorectal PC, either as separate treatments or in combination, depending on the preference of the center and surgeon.

The choice for HIPEC and/or EPIC has major consequences for the scheduling of procedures and the capacity of the treatment center. This becomes relevant as growing awareness among physicians about the availability of treatment possibilities for PC results in increasing numbers of patients being referred to specialized centers. 
No randomized controlled trials have been published comparing the survival outcomes after EPIC and HIPEC. Furthermore, it is unclear whether the combination of HIPEC and EPIC results in superior outcomes.

In a recent experimental study, it was shown that adjuvant application of HIPEC after CS can prolong survival as compared with CS only. ${ }^{6}$ A second study demonstrated beneficial results for both adjuvant normothermic and hyperthermic intraoperative perfusion. ${ }^{7}$

In the present study, the effectiveness of HIPEC and EPIC as adjuvant treatment after CS was evaluated in a well-established model for PC in rat. In addition, the combination of the two treatments was investigated. Survival is the primary outcome parameter.

\section{METHODS}

\section{Experimental Design}

PC was induced in all animals 7 days before the surgical procedures. Rats were assigned to one of four treatment groups $(n=20$ each) by randomization. Treatment per group was as follows: group 1 (CS), exploration followed by cytoreductive surgery only; group 2 (HIPEC), cytoreductive surgery plus hyperthermic intraperitoneal perfusion with mitomycin at $41^{\circ} \mathrm{C}$ for $90 \mathrm{~min}$; group 3 (EPIC), cytoreductive surgery followed by early postoperative chemotherapy from the first day postoperatively using mitomycin on the first day and 5-fluorouracil (5-FU) on days 2-5; group 4 (HIPEC+EPIC), cytoreductive surgery followed immediately by HIPEC and EPIC. Primary outcome parameter was survival.

\section{Animals}

Eighty male WAG/Rij rats (10-12 weeks old, mean weight $267 \pm 8.2 \mathrm{~g}$ ) were obtained from Harlan, Horst, The Netherlands. The animals were housed in filter-topped cages (three rats per cage) under clean, nonsterile standardized conditions (temperature $20-24^{\circ} \mathrm{C}$, relative humidity 50-60\%, $12 \mathrm{~h}$ light/12 h dark) with free access to water and chow (Ssniff; Bio services, Uden, The Netherlands). Accustomization to laboratory conditions was allowed for at least 1 week before the start of the experiment. All experiments were approved by the Animal Welfare Committee of Radboud University Nijmegen Medical Centre and carried out in accordance with the Dutch Animal Welfare Act 1997.

\section{Induction of Peritoneal Carcinomatosis}

Two milliliters of a suspension containing a concentration of $10^{6}$ cells $/ \mathrm{mL}$ of the syngeneic rat colon carcinoma cell line CC-531 was injected intraperitoneally in all animals as described elsewhere to induce peritoneal carcinomatosis. $^{8}$

\section{Surgery}

One week after tumor cell inoculation, CS was performed in all animals under general anesthesia using isoflurane $3 \%, \mathrm{O}_{2}$ and $\mathrm{N}_{2} \mathrm{O}$ 1:1. Carprofen $(5 \mathrm{mg} / \mathrm{kg} /$ day) was given for analgesia $30 \mathrm{~min}$ prior to surgery and once daily until the third postoperative day. To limit body heat loss, all rats were placed on a warmed mattress during the procedure.

The abdominal cavity was opened and exposed by complete midline laparotomy, and all abdominal regions were systematically inspected for tumor deposits. The peritoneal cancer index (PCI) representing the extent of peritoneal carcinomatosis was recorded as the sum of all scores, as described previously. ${ }^{6,7}$

All animals underwent CS with standard resection of the greater omentum aiming at radical removal of all macroscopic tumor deposits. CS may include resection of fat pads, spleen, parts of the mesentery, and peritonectomy. No bowel resections are performed in this experimental model, and no anastomoses are made. Due to the small size of the animals, some tumor localizations are impossible to reach while performing surgical resection. Small lesions that are not suitable for resection due to their localization are cauterized using an electrocoagulation device. These cauterized deposits are considered as completely treated, as no visible vital tumor tissue is left behind. After the best achievable cytoreduction, the amount of residual tumor was scored in accordance to current clinical practice using the R1-R2a-R2b classification: R1, no macroscopic disease left; R2a, tumor smaller than $2.5 \mathrm{~mm}$ left behind; R2b, residual tumor mass larger than $2.5 \mathrm{~mm}$.

In both the CS and EPIC groups the abdomen was closed. In the HIPEC and HIPEC+EPIC groups, surgery was followed immediately by heated intraperitoneal perfusion.

\section{HIPEC}

Perfusion of the abdomen was performed with a closed technique as described before. ${ }^{6,9}$ The peritoneal perfusate consisting of $250 \mathrm{~mL} \mathrm{NaCl}(0.9 \%)$ was warmed to $41.5^{\circ} \mathrm{C}$ and infused into the abdomen at $10 \mathrm{~mL} / \mathrm{min}$ during $90 \mathrm{~min}$. Mitomycin-C (Nycomed Christiaens BV, Breda, The Netherlands) was dissolved in saline and added to the perfusate in three separate gifts at $30 \mathrm{~min}$ intervals, each containing $50 \%, 25 \%$, and $25 \%$ of the total dose of $35 \mathrm{mg} / \mathrm{m}^{2}$ mitomycin. During the perfusion, gentle massage of the abdomen was applied to equalize fluid distribution in the 
peritoneal cavity. Subsequently the abdominal wall was closed in two layers using continuous Vicryl 3/0 sutures. All animals received subcutaneous injection of $10 \mathrm{~mL}$ $0.9 \% \mathrm{NaCl}$ for rehydration.

\section{EPIC}

Intraperitoneal chemotherapy was administered with a single daily intraperitoneal injection $(0.75 \mathrm{ml})$. On the first day following surgery, mitomycin was given at a dose of $10 \mathrm{mg} / \mathrm{m}^{2}(1.36 \mathrm{mg} / \mathrm{kg})$. From the second postoperative day onwards a dose of $15 \mathrm{mg} / \mathrm{kg} 5$-fluorouracil was given to each rat intraperitoneally. The last gift of 5-fluorouracil was administered on day 5 .

\section{Follow-up and Autopsy}

During the first week after surgery, general condition and body weight of the animals were assessed daily. After the first week, general condition and body weight were recorded at least twice weekly and more often if symptoms of discomfort were present. Survival was the primary endpoint of this study.

Humane endpoints were chosen to minimize or terminate pain or distress to the experimental animals via euthanasia rather than waiting for death as the endpoint. The following humane endpoints were used:

The animal refuses intake of food and fluids, shows rapid weight loss, severe circulation or breathing problems, strongly abnormal behavior, severe clinical symptoms or disabling consequences of ascites or tumor growth, or the expectation is raised that the animal will die shortly.

Whether these endpoints were reached was determined by an experienced biotechnician blinded to the animals' assigned treatment groups.

When the humane endpoint was reached, rats were killed by $\mathrm{O}_{2} / \mathrm{CO}_{2}$ asphyxiation and autopsied.

At autopsy, the weight of ascites (if present) was measured and the extent of intraperitoneal tumor load was recorded using the same PCI scoring system as described above. The experiment was terminated at 168 days postoperatively ( 24 weeks). The rats that were still alive at that time were euthanized and autopsied.

In case no macroscopic tumor deposits were found at autopsy, the greater omentum and diaphragm were removed and microscopically examined for presence of tumor growth. Hematoxylin and eosin (H\&E) staining and murine MG1 antibody in combination with a horse antimouse immunoglobulin $\mathrm{G}$ (IgG) antibody, horseradish peroxidase (HRP) conjugated (Vector Laboratories Inc., Burlingame, CA, USA) were used for microscopic evaluation of the samples.

\section{Statistical Analysis}

Statistical analysis was performed using Graphpad Prism (version 4.0, 2003; Graphpad Software Inc., San Diego, CA) and SPSS (version 17.0, 2007, Chicago, IL) software. One-way analysis of variance (ANOVA) testing was used for comparison of continuous values. The comparison of dichotomous values was performed with chisquare or Fisher's exact test.

Kaplan-Meier curves were used for survival analysis and compared by means of the log-rank test. To correct for confounding factors, Cox regression analysis was performed.

\section{RESULTS}

\section{Surgical Procedures}

All animals underwent cytoreductive surgery. No differences were observed between groups regarding preoperative clinical condition or mean body weight.

Macroscopic peritoneal carcinomatosis was present in all animals. An example of macroscopic tumor growth is shown in Fig. 1a. The greater omentum, perisplenic area, liver hilum, mesentery, and intra-abdominal site of tumor inoculation were the most commonly affected sites of tumor growth. Detailed PCI is given in Table 1. Median PCI before cytoreduction was similar in all treatment groups $(P=0.429)$.

No difference in results of CS in terms of residual disease were observed between the treatment groups $(P=0.343)$. Mean time of the CS procedures was 26 (standard deviation, SD 6) $\mathrm{min}$ without differences between groups $(P=0.221)$.

\section{Perfusion Characteristics}

Mean intra-abdominal temperature at the inflow site was $41.5^{\circ} \mathrm{C}$ (SD $0.44^{\circ} \mathrm{C}$ ). Mean rectal and intra-abdominal temperatures during perfusion are shown in Fig. 2. The perfusion characteristics were similar in both HIPEC and HIPEC+EPIC groups.

\section{Early Follow-up}

Figure 3 shows the course of body weight during the first 14 days after surgery in the four groups.

Rats in the CS group generally gained weight from postoperative day 2 onwards. In the HIPEC group, rats started gaining weight after day 6. Rats receiving EPIC (with or without HIPEC) reached their minimum weight on day 7. In both groups receiving EPIC, the maximum weight 

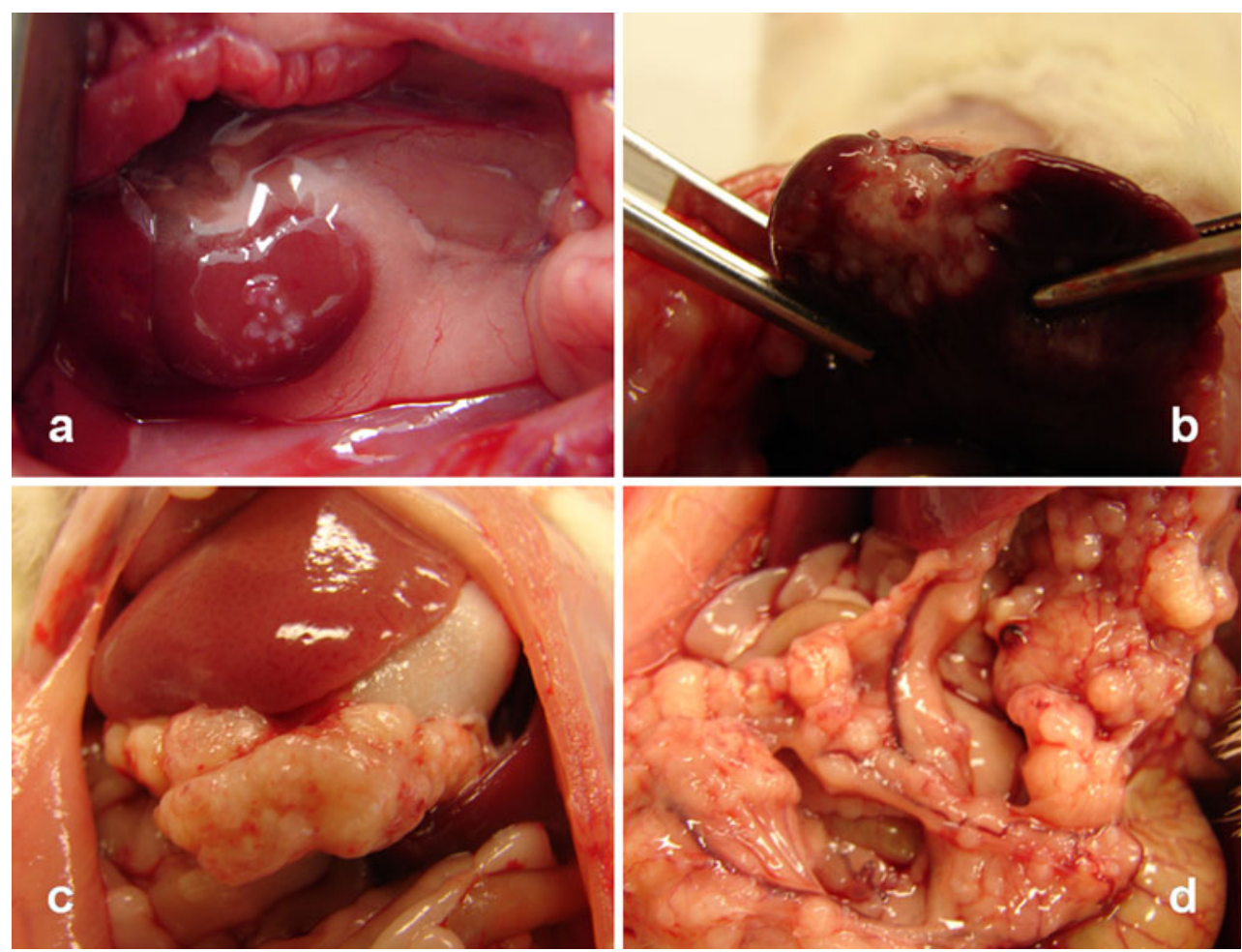

FIG. 1 Examples of macroscopic tumor deposits found during initial exploration of the abdomen on the kidney (a) and during autopsy at liver (b), omentum (c), and mesentery (d)

TABLE 1 Tumor score before cytoreduction and results of cytoreductive surgery
CS cytoreductive surgery, EPIC early postoperative intraperitoneal chemotherapy, HIPEC hyperthermic intraperitoneal chemotherapy, PCI peritoneal cancer index

\begin{tabular}{lllll}
\hline Group & CS & EPIC & HIPEC & HIPEC+EPIC \\
\hline $\begin{array}{l}\text { Preoperative weight (g), mean (SD) } \\
\text { Tumor score per site, median (range) }\end{array}$ & $267(7)$ & $265(9)$ & $268(9)$ & $267(8)$ \\
Subcutaneous & $1(0-2)$ & $1(0-1)$ & $1(0-1)$ & $1(0-2)$ \\
Inoculation site intra-abdominal & $1(0-3)$ & $1(0-3)$ & $1(0-2)$ & $1(0-3)$ \\
Greater omentum & $1(1-1)$ & $1(0-2)$ & $1(1-3)$ & $1(1-1)$ \\
Liver hilum & $1(0-1)$ & $1(0-1)$ & $1(1-3)$ & $1(1-2)$ \\
Liver surface & $0(0-0)$ & $0(0-0)$ & $0(0-1)$ & $0(0-0)$ \\
Spleen & $1(0-1)$ & $1(0-1)$ & $1(1-2)$ & $1(0-2)$ \\
Mesentery & $1(1-3)$ & $1(0-3)$ & $1(1-2)$ & $1(0-3)$ \\
Fat pad left & $0(0-1)$ & $0(0-1)$ & $0.5(0-1)$ & $0(0-1)$ \\
Fat pad right & $0(0-2)$ & $0.5(0-2)$ & $1(0-2)$ & $0(0-2)$ \\
Diaphragm & $0(0-2)$ & $0(0-1)$ & $0(0-1)$ & $0(0-2)$ \\
Parietal peritoneum & $0(0-3)$ & $0(0-2)$ & $0(0-3)$ & $0(0-3)$ \\
PCI, mean (SD) & $8.0(2.9)$ & $7.3(2.0)$ & $8.3(1.8)$ & $7.4(2.0)$ \\
Splenectomy $(n)$ & 1 & 1 & & 3 \\
Yes & 19 & 19 & 17 & 17 \\
No & 16 & 19 & 18 & 18 \\
Completeness of resection $(n)$ & 2 & 1 & 2 & 2 \\
R1 & 2 & 0 & 0 & 0 \\
R2a & & & & \\
R2b & 16 & & \\
\hline
\end{tabular}




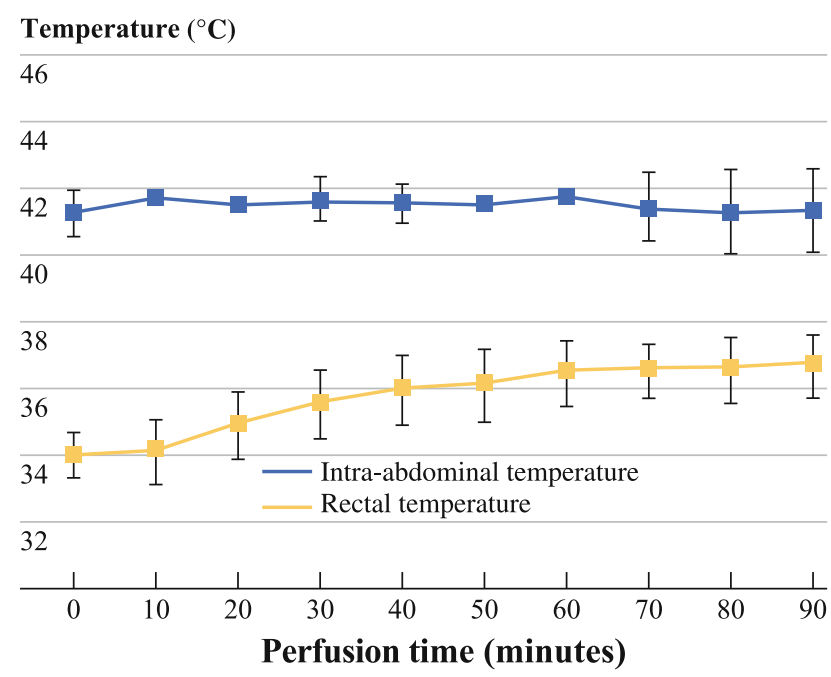

FIG. 2 Rectal and intra-abdominal temperatures during perfusion procedures. Data represent mean $\pm \mathrm{SD}(n=40)$ of temperature measurements during HIPEC in all animals from both HIPEC and HIPEC+EPIC groups

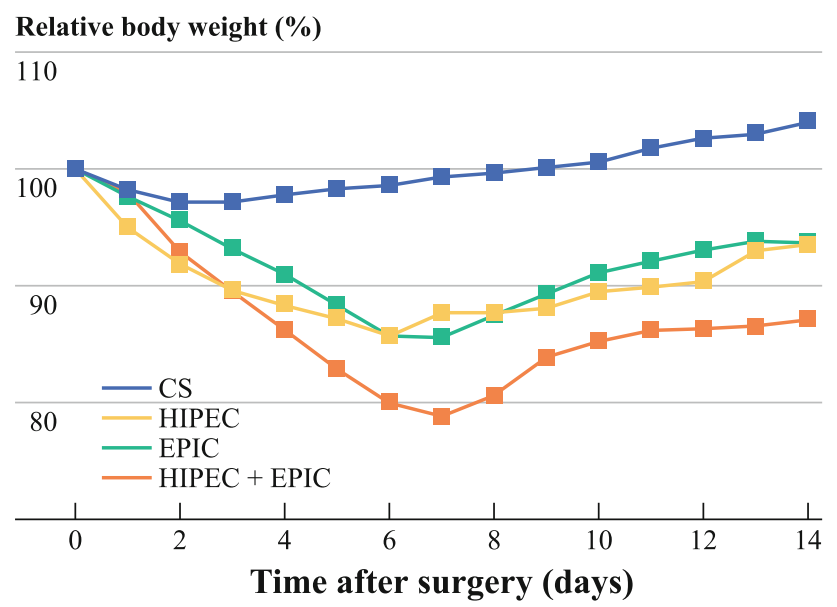

FIG. 3 Postoperative course of mean relative body weight

loss was higher than in the CS group (CS versus HIPEC, $P=0.056$; CS versus EPIC and CS versus HIPEC+EPIC, $P<0.001)$.

Eight deaths occurred in the early postoperative course (two HIPEC, three HIPEC+EPIC, two CS, and one EPIC) unrelated to tumor growth. In the CS group, one rat died of postoperative ileus after 4 days. The other rat died of unknown reasons after 3 days. In the EPIC group, one rat died after 12 days because of obstruction caused by herniation of the ileum through a mesentery defect. In the HIPEC group, two unexpected deaths occurred, one after 7 days because of an abscess in the upper abdomen, and one caused by respiratory failure immediately following the surgical procedure, possibly related to excessive blood loss during CS. In the HIPEC+EPIC group, deaths were

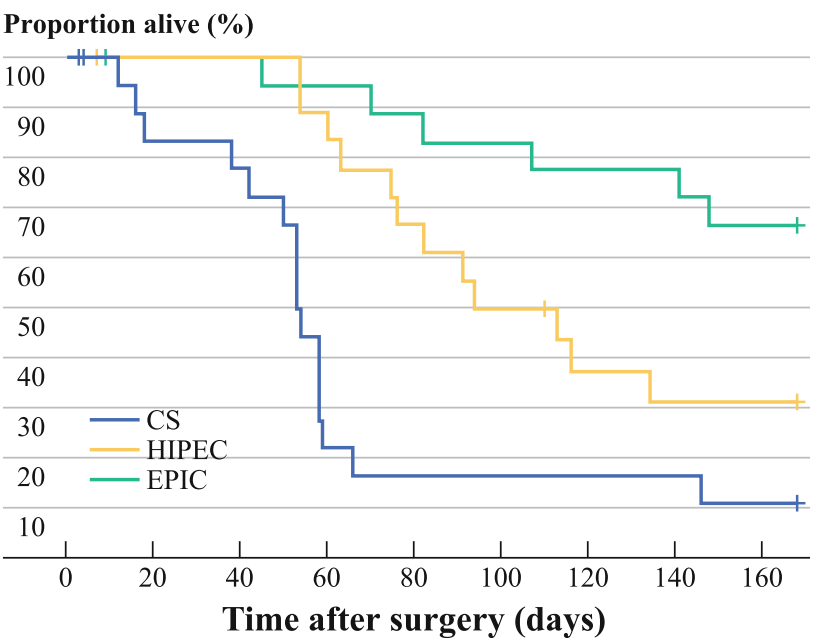

FIG. 4 Kaplan-Meier analysis of overall survival, per group

caused by bowel perforation and necrosis after 2 days and abscess formation in the liver hilum resulting in obstruction and progressive icterus after 15 days. One rat was found dead after 8 days, and the cause of death remained unclear.

In the HIPEC+EPIC group, a late unexpected death occurred after 86 days. At autopsy, herniation of the small bowel was found. All rates mentioned above were marked as censored in the survival analysis.

Rats in the HIPEC+EPIC group initially recovered from the procedures and gained weight from day 8 onwards. However, after several weeks rats started to lose weight again to die eventually from excessive weight loss. This course was observed in 11 rats. At autopsy, no tumor was found in these animals, nor any evidence for another cause of death. Only four rats died from the consequences of tumor growth. At the end of the experiment, two rats were alive. Given the large number of rats dying from nontumor-related causes in this group, the effect of treatment on survival could not be evaluated, and therefore it was decided not to include this group in the survival analysis.

\section{Survival and Post Mortem Findings}

Figure 4 shows the Kaplan-Meier survival analysis for each group. Median survival in the group treated with CS only was 53 days (95\% confidence interval (CI) 49-57 days). In the group of rats receiving HIPEC perfusion, median survival of 94 days (95\% CI 51-137 days) was observed. In the EPIC group, 12 animals were still alive when the experiment was ended and therefore median survival could not be recorded, but it was at least 168 days.

Survival outcomes were compared between groups, correcting for completeness of resection and preoperative PCI. Survival outcomes in both adjuvant treatment groups were significantly better as compared with the CS group: 
TABLE 2 Post mortem findings

\begin{tabular}{llllr}
\hline Group & CS & EPIC & HIPEC & $P$-value \\
\hline PCI, mean (SD) & $27(4)$ & $14(10)$ & $19(6)$ & $<0.001$ \\
Ascites weight (g), mean (SD) & $27(23)$ & $40(26)$ & $43(20)$ & 0.136
\end{tabular}

$C S$ cytoreductive surgery, EPIC early postoperative intraperitoneal chemotherapy, HIPEC hyperthermic intraperitoneal chemotherapy, $P C I$ peritoneal cancer index

HIPEC versus CS $(P=0.001$, hazard ratio for dying 0.24 , 95\% CI $0.10-0.55)$ and EPIC versus CS $(P<0.001$, hazard ratio $0.10,95 \%$ CI $0.04-0.28$ ).

Nineteen rats were still alive at the time of maximum follow-up of 168 days. At autopsy 11 of these rats appeared to be macroscopically free of tumor and ascites (1/2 CS, 3/5 HIPEC, 7/12 EPIC). In all of these rats, the remaining omentum and diaphragm were removed and stained for histological examination. No microscopic evidence of disease was found.

Rats from the CS group that reached their humane endpoint during follow-up showed a higher mean PCI at autopsy than rats treated with HIPEC $(P=0.004)$ or EPIC $(P<0.001)$. The differences in the amount of ascites present at autopsy remained statistically insignificant for both adjuvant treatment groups as compared with the CS group. Post mortem findings are summarized in Table 2.

On multivariate analysis, the influence of PCI and completeness of resection on survival outcomes did not reach significance ( $P=0.065$ and $P=0.164$, respectively).

\section{DISCUSSION}

In this experimental study, both EPIC and HIPEC were effective in prolonging survival when applied as adjuvant treatment after CS. Always keeping in mind the difference between rodents and humans, this finding may have important implications for clinical practice.

The scheduling of EPIC treatment has several logistical advantages when compared with HIPEC. For the performance of a HIPEC procedure, careful and punctual scheduling of the surgical procedure is essential. Intraoperative administration of heated chemotherapy requires the availability of the chemotherapeutic agent in the operating room, safety precautions for both the patient and the personnel involved in the procedures, specialized technical equipment which is adequately cleaned and/or sterilized, and the presence of an experienced perfusionist. In contrast, for administration of EPIC, insertion of a peritoneal port at the end of a surgical procedure is an easier and less time-consuming procedure. The intraperitoneal treatment itself can be performed at an intensive care unit or even hospital ward. Furthermore, EPIC can be offered ad hoc to patients in whom peritoneal carcinomatosis is discovered incidentally during laparotomy, which is a common way of diagnosing this disease. However, performance of EPIC has been associated with an increased risk of complications after extensive abdominal surgery in some retrospective studies. ${ }^{10,11}$ This theoretically may be a consequence of the prolonged contact of chemotherapy with newly performed anastomoses and operated surfaces, thereby delaying wound healing and recovery. Indeed, experimental studies have shown that both HIPEC and intraperitoneal injection of chemotherapy have a negative influence on wound strength. ${ }^{12,13}$

Other disadvantages of EPIC may be a less equal distribution of fluid in the abdomen and patients experiencing physical discomfort (nausea, impaired mobility) associated with the intra-abdominal presence of the chemotherapy for 5 days. However, other studies reported no differences in complications between HIPEC and EPIC. ${ }^{14,15}$ To date, no randomized controlled trials have been reported comparing the two techniques in this respect.

Previously published animal experiments have shown that the timing of intraperitoneal chemotherapy treatment after tumor cell application is a relevant factor influencing the effectiveness of treatment. It has been described that administration of anticancer agents immediately after tumor cell inoculation can eliminate all disease, whereas late administration (later than 3 days after tumor inoculation, when usually macroscopic disease is present) may slow down tumor growth but cannot be used with curative intent. ${ }^{16-20}$ The current study shows, in accordance with the experimental literature mentioned, that the application of intraperitoneal chemotherapy 1 day after surgery is still effective to eliminate microscopic tumor cells after cytoreductive procedures, without use of hyperthermic circumstances. Twelve animals from the EPIC group and five animals from the HIPEC group were alive at the end of the follow-up period of 24 weeks. Three animals from the HIPEC and seven from the EPIC group were free of microscopic tumor.

Due to the experimental design of this study, choices such as the HIPEC conditions applied had to be made, which always constitute a certain degree of bias. Although the flow rate in rats may be lower than in the human situation, it is thought unlikely that this would explain the survival outcomes of this study. To our knowledge, the flow rate is not a commonly reported prognostic factor in patients treated with HIPEC. In the rats receiving EPIC, chemotherapy was given as a low-volume intraperitoneal injection, resulting in no flow or pressure at all.

A direct cytotoxic effect of higher temperatures (exceeding $42-43^{\circ} \mathrm{C}$ ) on both normal and tumor cells has been observed under experimental conditions. ${ }^{21,22}$ In HIPEC treatment, however, direct cytotoxicity by hyperthermia is not a primary goal. The hyperthermia applied in this 
treatment is aimed at a synergetic effect with chemotherapy. Therefore, in this animal model, lower temperatures are used, based on the HIPEC technique that was described in the only completed randomized phase III trial evaluating the effectiveness of HIPEC using an inflow temperature of $41-42^{\circ} \mathrm{C} .{ }^{1}$

The necessity of hyperthermia in the treatment of peritoneal carcinomatosis has never been proven.

Although mild hyperthermia as applied in HIPEC is thought to increase blood flow and oxygen content within tumors, thereby increasing drug concentrations and enhancing the antitumor effect, the presumed beneficial effect on patient survival has never been investigated in randomized trials. $^{22,23}$ In retrospective studies, no difference between normothermic and hyperthermic administration of mitomycin followed by EPIC was found. ${ }^{24}$ In a recent experimental study, normothermic and hyperthermic intraoperative perfusion were both effective in prolonging overall survival in rats with peritoneal carcinomatosis, and with both treatments several animals remained free of disease until the end of the follow-up period. ${ }^{7}$ In a study by Zeamari et al., similar results were obtained, although no cytoreductive surgery was performed. ${ }^{25}$ Also in the current study, the performance of EPIC (under normothermic conditions) was shown to be at least equally effective as the treatment including hyperthermia. Therefore, further research into the necessity of hyperthermia should be performed to investigate if indeed simplification of the current multimodality treatment for peritoneal carcinomatosis is possible.

In theory, EPIC and HIPEC may be combined to obtain optimal oncologic outcome. However, a risk of toxicity of the high dose of chemotherapy should always be taken into account when considering the combination of treatments. In the current study most rats treated with HIPEC+EPIC suffered from severe toxicity as illustrated by the weight loss during the first two postoperative weeks. In the end most rats died after several weeks with symptoms suggesting late toxicity. For further evaluation of the combination of HIPEC and EPIC in experimental studies, it may be useful to use only 5-FU in the EPIC regimen in order to decrease toxicity.

In conclusion, both HIPEC and EPIC prolonged survival when used as adjuvant therapy after cytoreductive surgery for peritoneal carcinomatosis from colorectal cancer in an experimental model. Further research is required to evaluate and compare the possible beneficial and adverse effects associated with both treatments in daily clinical practice. In this way the optimal treatment strategy for patients suffering from peritoneal carcinomatosis of colorectal origin will be further improved.

\section{Conflict of interest None.}

Open Access This article is distributed under the terms of the Creative Commons Attribution Noncommercial License which permits any noncommercial use, distribution, and reproduction in any medium, provided the original author(s) and source are credited.

\section{REFERENCES}

1. Verwaal VJ, van Ruth S, de Bree E, van Sloothen GW, van Tinteren $\mathrm{H}$, Boot $\mathrm{H}$, et al. Randomized trial of cytoreduction and hyperthermic intraperitoneal chemotherapy versus systemic chemotherapy and palliative surgery in patients with peritoneal carcinomatosis of colorectal cancer. J Clin Oncol. 2003;21: 3737-43.

2. Verwaal VJ, van Ruth S, Witkamp A, Boot H, van Slooten G, Zoetmulder FA. Long-term survival of peritoneal carcinomatosis of colorectal origin. Ann Surg Oncol. 2005;12:65-71.

3. Jayne DG, Fook S, Loi C, Seow-Choen F. Peritoneal carcinomatosis from colorectal cancer. Br J Surg. 2002;89:1545-50.

4. Sadeghi B, Arvieux C, Glehen O, et al. Peritoneal carcinomatosis from non-gynecologic malignancies: results of the EVOCAPE 1 multicentric prospective study. Cancer. 2000;88:358-63.

5. Lemmens VEPP, Klaver YLB, Verwaal VJ, Rutten HJT, Coebergh JWW, De Hingh IHJT. Predictors and survival of synchronous peritoneal carcinomatosis of colorectal origin: a population-based study. Int J Cancer. 2011;128:2717-25.

6. Klaver YLB, Hendriks T, Lomme RMLM, Rutten HJT, Bleichrodt RP, De Hingh IHJT. Intraoperative hyperthermic intraperitoneal chemotherapy after cytoreductive surgery for peritoneal carcinomatosis in an experimental model. Br J Surg. 2010;97:1874-80.

7. Klaver YLB, Hendriks T, Lomme RMLM, Rutten HJT, Bleichrodt RP, De Hingh IHJT. Hyperthermia and intraperitoneal chemotherapy for the treatment of peritoneal carcinomatosis: an experimental study. Ann Surg. 2011;254:125-30.

8. Lopes Cardozo AM, Gupta A, Koppe MJ, Meijer S, van Leeuwen PA, Beelen RJ, et al. Metastatic pattern of CC531 colon carcinoma cells in the abdominal cavity: an experimental model of peritoneal carcinomatosis in rats. Eur J Surg Oncol. 2001;27: 359-63.

9. Aarts F, Hendriks T, Boerman OC, Koppe MJ, Oyen WJ, Bleichrodt RP. A comparison between radioimmunotherapy and hyperthermic intraperitoneal chemotherapy for the treatment of peritoneal carcinomatosis of colonic origin in rats. Ann Surg Oncol. 2007;14:3274-82.

10. Glehen O, Kwiatkowski F, Sugarbaker PH, et al. Cytoreductive surgery combined with perioperative intraperitoneal chemotherapy for the management of peritoneal carcinomatosis from colorectal cancer: a multi-institutional study. J Clin Oncol. 2004; 22:3284-92.

11. Elias D, Benizri E, DiPietrantonio D, Menegon P, Malka D, Raynard B. Comparison of two kinds of intraperitoneal chemotherapy following complete cytoreductive surgery of colorectal peritoneal carcinomatosis. Ann Surg Oncol. 2007;14:509-14.

12. van der Kolk BM, de Man BM, Wobbes T, Hendriks T. Is early post-operative treatment with 5-fluorouracil possible without affecting anastomotic strength in the intestine? Br J Cancer. 1999; 79:545-50.

13. Aarts F, Bleichrodt RP, de Man BM, Lomme RMLM, Boerman OC, Hendriks T. The effects of adjuvant experimental radioimmunotherapy and hyperthermic intraperitoneal chemotherapy on intestinal and abdominal healing after cytoreductive surgery for peritoneal carcinomatosis in the rat. Ann Surg Oncol. 2008;15: 3299-307. 
14. Gomez PA, Barrios P, Rufian S, et al. Management of peritoneal surface malignancy with cytoreductive surgery and perioperative intraperitoneal chemotherapy. Eur J Surg Oncol. 2006;32: 628-31.

15. Elias D, Blot F, El Otmany A, et al. Curative treatment of peritoneal carcinomatosis arising from colorectal cancer by complete resection and intraperitoneal chemotherapy. Cancer. 2001;92:7176.

16. Hribaschek A, Pross M, Kuhn R, et al. Prevention and treatment of peritoneal carcinomatosis in experimental investigations with CPT-11 and oxaliplatin. Anticancer Drugs. 2002;13:605-14.

17. Hribaschek A, Meyer F, Schneider-Stock R, Pross M, Ridwelski $\mathrm{K}$, Lippert H. Comparison of intraperitoneal with intravenous administration of taxol in experimental peritoneal carcinomatosis. Chemotherapy. 2007;53:410-417.

18. Jacquet P, Stuart OA, Dalton R, Chang D, Sugarbaker PH. Effect of intraperitoneal chemotherapy and fibrinolytic therapy on tumor implantation in wound sites. J Surg Oncol. 1996;62:128-34.

19. Duvillard C, Benoit L, Moretto P, et al. Epinephrine enhances penetration and anti-cancer activity of local cisplatin on rat subcutaneous and peritoneal tumors. Int J Cancer. 1999;81:779-84.
20. Monneuse O, Mestrallet JP, Quash G, Gilly FN, Glehen O. Intraperitoneal treatment with dimethylthioampal (DIMATE) combined with surgical debulking is effective for experimental peritoneal carcinomatosis in a rat model. J Gastrointest Surg. 2005;9:769-74.

21. Hildebrandt B, Wust P. The biologic rationale of hyperthermia. Cancer Treat Res. 2007;134:171-84.

22. Song CW, Park HJ, Lee CK, Griffin R. Implications of increased tumor blood flow and oxygenation caused by mild temperature hyperthermia in tumor treatment. Int J Hyperth. 2005;21:761-67.

23. Hildebrandt $B$, Wust $P$, Ahlers $O$, et al. The cellular and molecular basis of hyperthermia. Crit Rev Oncol Hematol. 2002; 43:33-56.

24. da Silva RG, Sugarbaker PH. Analysis of prognostic factors in seventy patients having a complete cytoreduction plus perioperative intraperitoneal chemotherapy for carcinomatosis from colorectal cancer. J Am Coll Surg. 2006;203:878-86.

25. Zeamari S, Floot B, van der Vange N, Stewart FA. Pharmacokinetics and pharmacodynamics of cisplatin after intraoperative hyperthermic intraperitoneal chemoperfusion (HIPEC). Anticancer Res. 2003;23:1643-48. 